\title{
Phenol Injection versus Depomedrol Injection in Sacroiliac Joint Pain
}

\author{
Khalid Ali Khaleel ${ }^{1}$, Zaid Al-Attar ${ }^{2 *}$
}

${ }^{1} \mathrm{MBChB}$, DA FICMS Senior anesthesiologist in Dept. of Anesthesia, Intensive Care and Pain Management, Neuroscience hospital / Baghdad /Iraq

${ }^{2} \mathrm{MBChB}, \mathrm{PhD}$ (Pharmacology) Lecturer, Dept. of pharmacology, Al-Kindy College of Medicine / University of Baghdad /Baghdad /Iraq

DOI: $10.36347 /$ sajp.2020.v09i03.003

| Received: 17.03.2020 | Accepted: 24.03.2020 | Published: 27.03.2020

*Corresponding author: Zaid Al-Attar

Abstract

Original Research Article

Objective: Our purpose is to assess the effectiveness of phenol $7 \%$ in aqueous solution for neurolysis versus depomedrol $80 \mathrm{mg}$ injection in patients with severe chronic nonmalignant sacroiliac joint pain syndrome who did not achieve adequate pain control (visual analog scale [VAS] 3) with conventional pain treatment. Design: A hundred patients with severe nonmalignant sacroiliac joint pain persisting for 6 months or longer were followed for more than 6 months after phenol neurolysis in this prospective observational study. All patients had previously received narcotic drugs, with or without nonsteroidal anti-inflammatory agents or adjuvants, without adequate pain relief. An aqueous solution of phenol $7 \%$ was used for chemical neurolysis. Sacroiliac joint injection was done in theatre under ultrasound guidance using linear probe. Results: Good pain relief (VAS $\leq 3$ ) was achieved in 35 patients after neurolysis with phenol, and the mean VAS decreased from $8.74 \pm 1.08$ (range 7-10) before treatment to $1.93 \pm$ 2.41 after treatment $(\mathrm{P}<0.0001)$. Lower backache of lower Oswestry disability index (ODI) shows a lowering effect of phenol as compared with depomedrol at 3 years' time interval $p<0.001$. Conclusion: The use of phenol $7 \%$ in aqueous solution is an effective and safe technique for neurolysis. Because of the potential risk of flaccid paralysis, this technique should be used in selected cases, far removed from motor nerves and the spinal cord.

Keywords: Chronic Nonmalignant Pain; Phenol; Neurolysis.

Copyright @ 2020: This is an open-access article distributed under the terms of the Creative Commons Attribution license which permits unrestricted use, distribution, and reproduction in any medium for non-commercial use (NonCommercial, or CC-BY-NC) provided the original author and source are credited.

\section{INTRODUCTION}

Pain is the most common reason for patients to seek medical care. Chronic pain is associated with a considerable economic and social burden. About 50 million people experience chronic pain in the United States [1], and the associated total cost varies from $\$ 80$ to $\$ 100$ billion in direct and indirect expenses from medical care, lost income, and decreased productivity in work. The successful treatment of chronic pain has obvious economic and social significance. The World Health Organization established a "three-step analgesic ladder" guideline for cancer pain treatment, in which drugs with the fewest side effects and the least potential for addiction are prescribed first. This approach can be adopted for non-malignant chronic pain as well [2], and interventional procedures were adopted as the fourth step.

Sometimes conventional therapeutic measures fail to optimize pain control and additional techniques are required to achieve enhanced functional capacity, physical, and psychological well-being, and enhanced quality of life for patients with chronic pain. In these cases, chemical (phenol or alcohol) or physical (cryotherapy, thermocoagulation, or radiofrequency) neurodestructive techniques can be used. In recent years, radiofrequency neuroablation has gained popularity for the treatment of most common chronic nonmalignant pain syndromes [3]. However, radiofrequency equipment is very expensive. For institutions that cannot afford this equipment, chemical neurolysis can be an effective alternative for patients with severe chronic non-malignant pain unresponsive to conventional therapy.

The most important sequel of sacroiliac joint injection of depomedrol is recurrence of pain, so in this study we investigate if phenol $7 \%$ instead can reduce the rate of recurrence.

The purpose of this report is to present our experience with phenol neurolysis in patients with several severe chronic pain syndromes unresponsive to conventional modalities of treatment.

\section{MATERIALS AND METHODS}

Study was done in neurosciences and medical city hospital in period extended from January 2010 to 
July 2018 on 100 patients, 60 patients are females, 40 patients are males. Age of patients ranged between 25 to 65 year. Patients who have infection at site of injection, patients with coagulopathy, diabetic patients are excluded from the study. Patients were divided into two groups each with 50 patients. $1^{\text {st }}$ group was given depomedrol injection $80 \mathrm{mg}$ in $2 \mathrm{ml}$ into sacroiliac joint. The second group was given $7 \%$ phenol $1 \mathrm{ml}$ into the same joint. After patient's selection and subdivision into 2 groups using sealed subdivision envelope method, patients were informed about the procedure results and possible complications. They signed the interventional consent and admitted to theatre. IV line was inserted under full monitoring of PR, BP, SPO2 in prone position under aseptic technique. Diagnostic test was done by using lignocaine $2 \mathrm{ml}$ injected in SI joint to see if pain subside or abolish after injection. 70 percent reduction in VAS regarded as positive, injection was done under ultrasound guidance using linear probe ezona 3000 machine.

If diagnostic test was positive then we inject either depomedrol or phenol accordingly, then, patients were discharged from hospital on the same day.

Then, they were followed for 36 months and assessed by using VAS (visual analogue scale), lower backache of lower Oswestry disability index (ODI).

Statistical analysis was conducted using graph pad prism software and ANOVA test.

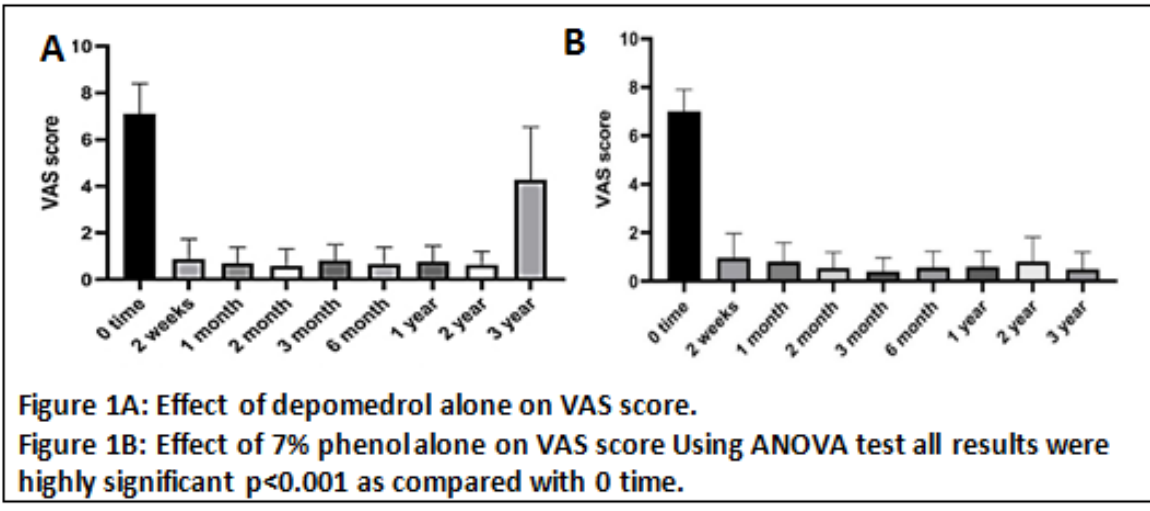

\section{RESULTS}

The results of 50 patients suffering from sacroiliac pain given depomedrol shows that depomedrol is highly effective in relieving sacroiliac pain with high significance at all time intervals (figure 1A).

Another group of patients suffering from sacroiliac pain were given $7 \%$ phenol injection over the same time intervals and VAS score was recorded for them (figure 1B). Phenol is highly effective in relieving sacroiliac pain with high significance at all time intervals.
Then, by comparing the results of depomedrol and phenol in one graph (figure 2), we can notice that VAS scores of depomedrol and phenol are very close with no significant difference except at 3 years interval when phenol is much better than depomedrol with high statistical significance $\mathrm{p}<.001$.

Lower backache of lower Oswestry disability index (ODI) shows a lowering effect of both depomedrol and phenol (figures $3 \mathrm{~A}$ and $\mathrm{B}$ ). However, phenol shows a statistical significance as compared with depomedrol at 3 years' time interval $\mathrm{p}<0.001$ (figure 4). 
No treatment-related serious complications, such as flaccid paralysis or tissue necrosis, were observed. We registered, however, minor complications such as local hematoma in three patients, and pain at the injection site that disappeared after less than 2 weeks in six patients.
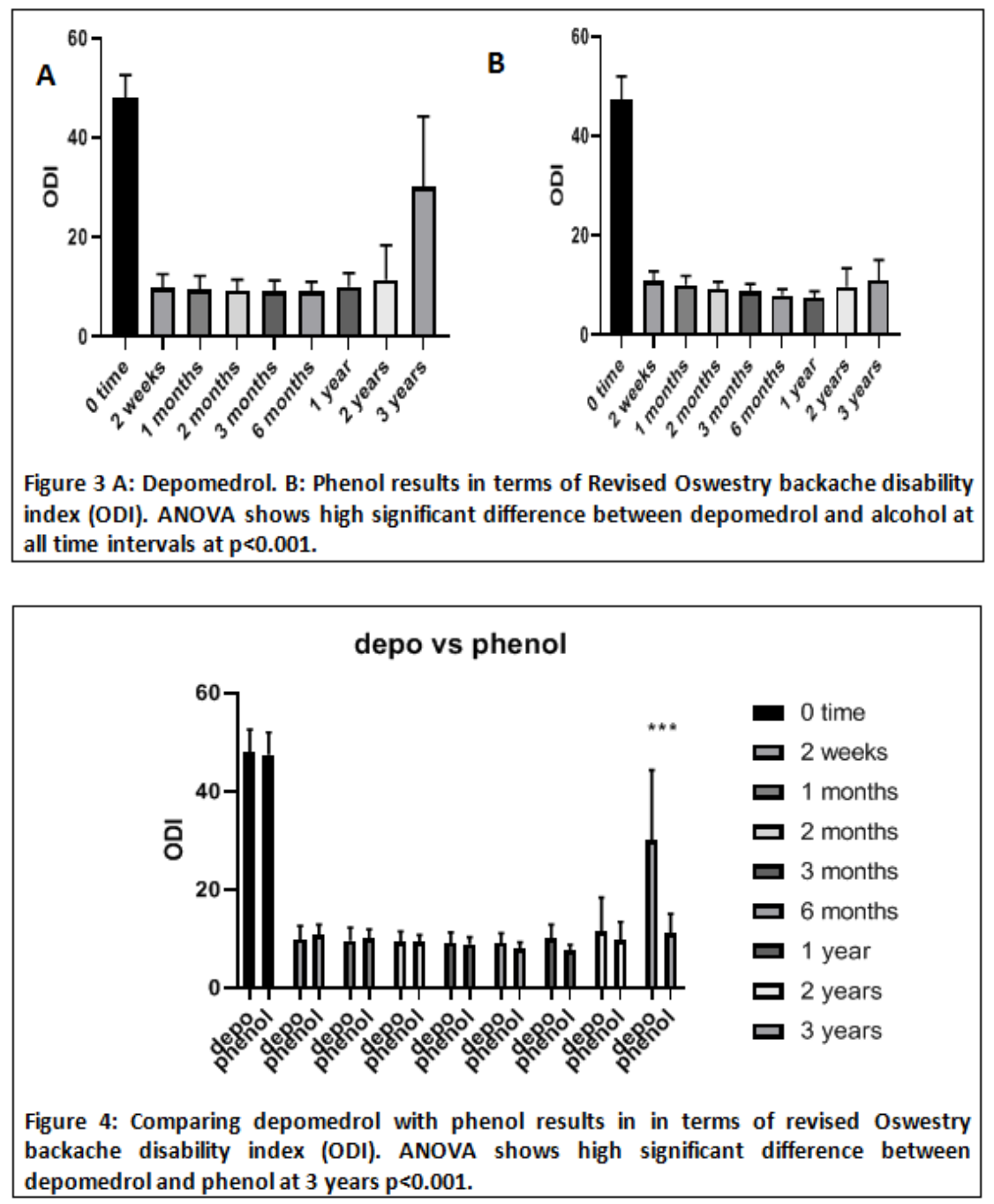

\section{DISCUSSION}

The use of phenol for neuroablation is widely accepted in chronic malignant pain, especially when life expectancy is low. However, the use of chemical neurolysis for chronic nonmalignant pain is less common. Reluctance to use phenol for chronic nonmalignant pain stems from the fear of inducing severe neural injury and physical incapacity in patients with a normal life expectancy [4]. Nevertheless, the American Society of Anesthesiologists Task Force on Pain Management, Chronic Pain Section, recommends the use of chemical (phenol or alcohol) or thermal (radiofrequency or cryoneurolysis) neurolysis if other techniques have failed to provide adequate pain control.

Phenol sympatholysis is a recognized technique for the treatment of advanced vascular disease [5], Raynaud's disease, and hyperhidrosis. Phenol is also useful for postoperative analgesia and for prolonged analgesia after trauma or pos-therapeutic neuralgia. There were no neurological complications in one report of 3,485 patients who underwent intercostal neurolysis[6].

In the present series, neurolysis with phenol was very effective in the treatment of groin pain due to genitofemoral nerve entrapment following inguinal hernia repair, with adequate and longlasting pain relief (more than 6 months) in three patients with this condition. Similar results were observed in two patients suffering from Arnold's (greater occipital nerve) neuralgia. Phenol infiltration has been recommended as an alternative therapy when surgical treatment for painful neuromas fails [6]. This treatment induced a long pain-free period in one of our patients who had a painful neuroma after partial amputation. We believe that phenol neurolysis can effectively and safely replace surgical neurolysis for these chronic pain syndromes. 
The use of phenol neurolysis is not without risk. When injected near motor nerves, phenol can produce flaccid paralysis and might also cause systemic complications, such as nausea and vomiting, central nervous system stimulation, cardiovascular depression, and cardiac arrhythmias. Phenol doses less than $100 \mathrm{mg}$ seem not cause serious systemic side effects. Therefore, it must be used only after proper selection of patients and with adequate positioning of the needle under imaging[7].

Chemical neuritis and severe burning pain in the distribution of the nerve as reported after phenol neurolysis, but we did not observe this complication in any patient of this series. We speculate that the absence of this complication can be the result of the small sample size of this observation. More studies based on large series should be undertaken in order to evaluate its incidence.

The ideal concentration of phenol for neurolytic treatment is not well determined and varies from $3 \%$ to $12 \%$. It is well known that aqueous solution is more potent than glycerin and the addition of small amounts of glycerol or water soluble radio-opaque contrast material may increase the concentration to $15 \%[8]$.

Ethyl alcohol is a potent drug and can also be used to produce neurolysis. We prefer to inject phenol because alcohol produces severe burning pain on injection and both drugs have similar efficacy[9].

Phenol for SI joint is a great idea, but concerned about leakage, Fortin's SI joint local anesthesia injection study reported that $40 \%$ of the studied population (asymptomatic volunteers) reported lower extremity numbness after injection. A large percentage of these people have articular inflammation or irritation of pain fibers at the very inner margin of the joint capsule. Otherwise, the LA could not provide excellent pain relief so quickly.
Sacroiliac joint has dual nerve supply the posterior part supplied by sensory branches from dorsal rami of sacral nerves $\mathrm{S} 1$ to $\mathrm{S} 1$ while anterior part supplied by sensory branches from ventral rami of S1 to S5 so blocking of posterior part of the joint is not enough to relieve pain because the anterior part is still not blocked. During radio frequency ablation the needle cannot reach the anterior part it reach only the posterior part so there is incomplete pain relief while in phenol ablation the solution supposed to reach the anterior part that is why the rate of recurrence is higher in radio frequency than phenol ablation.

\section{REFERENCES}

1. Katz WA. Musculoskeletal pain and its socioeconomic implications. Clinical rheumatology. 2002;21 Suppl 1:S2-4.

2. Russo CM, Brose WG. Chronic pain. Annu Rev Med. 1998;49:123-33.

3. Van Zundert J, Raj P, Erdine S, van Kleef M. Application of radiofrequency treatment in practical pain management: state of the art. Pain Pract. 2002;2(3):269-78.

4. Colloca L, Ludman T, Bouhassira D, Baron R, Dickenson AH, Yarnitsky D. Neuropathic pain. Nat Rev Dis Primers. 2017;3:17002.

5. Rosen RJ, Miller DL, Imparato AM, Riles TS. Percutaneous phenol sympathectomy in advanced vascular disease. AJR Am J Roentgenol. 1983;141(3):597-600.

6. Weksler N, Klein M, Gurevitch B, Rozentsveig V, Rudich Z, Brill S. Phenol Neurolysis for Severe Chronic Nonmalignant Pain: Is the Old also Obsolete? Pain Medicine. 2007;8(4):332-7.

7. Candido K, Stevens RA. Intrathecal neurolytic blocks for the relief of cancer pain. Best Pract Res Clin Anaesthesiol. 2003;17(3):407-28.

8. Baheti DK, Bakshi S, Gehdoo RP, Gupta S. Interventional Pain Management: A Practical Approach: Jaypee Brothers, Medical Publishers Pvt. Limited; 2016.

9. Niesel HC, Klimpel L, Kaiser H, al-Rafai S. [The single intercostal block--surgical and therapeutic indications]. Reg Anaesth. 1989;12(1):1-12. 